Connotas. Revista de crítica y teoría literarias 


\begin{tabular}{|ll|}
\hline PQ6001 & Connotas. Revista de crítica y teoría literarias / \\
Director Fortino Corral Rodríguez.-Hermosillo, Sonora: \\
unIson. Departamento de Letras y Lingüística. \\
C2011. \\
V-: $23 \mathrm{~cm}$. \\
Semianual \\
Año VII, No. $12(2011)$ \\
ISSN: 1870-6630 \\
Incluye bibliografía. \\
1. Literatura española - Historia y crítica-Publicaciones \\
periódicas. 2. Literatura hispanoamericana - Publicaciones \\
periódicas. I. Corral Rodríguez, Fortino, dir.
\end{tabular}

CONNOTAS. REVISTA DE CRÍTICA Y TEORÍA LITERARIAS. Año VII, núm. 12, enero junio 2011, es una publicación semestral editada por la Universidad de Sonora, a través de la División de Humanidades y Bellas Artes, en el Departamento de Letras y Lingüística. Blvd. Luis Encinas y Blvd. Rosales s/n, Col. Centro, C.P. 83000, Hermosillo Sonora; Tel. (662) 2592 136, (662) 2592 157, <www.uson.mx>, <http://www.connotas.uson.mx, connotas@capomo.uson.mx>. Editor responsable: Rosario Fortino Corral Rodríguez. Reservas de Derechos al Uso Exclusivo núm. 04-2006-020714184900-102. ISSN: 1870 6630; ambos otorgados por el Instituto Nacional del Derecho de Autor. Licitud de Título núm. 13434 y de Contenido núm. 11007, otorgados por la Comisión Calificadora de Publicaciones y Revistas Ilustradas de la Secretaría de Gobernación. Impresa en Impresos RM S.A. de C.V., Privada Miguel Alemán no. 17, col. San Benito, Hermosillo, Sonora, México. Tel. 2 100212, e-mail impresosrm@gmail.com. Este número se terminó de imprimir el 5 de octubre de 2011 con un tiraje de 300 ejemplares. Las opiniones expresadas por los autores no necesariamente reflejan la postura del editor de la publicación.

Se autoriza la reproducción total o parcial de los contenidos de la presente publicación, siempre y cuando se acredite adecuadamente el origen de los mismos.

Este número se publicó con apoyo del Programa Integral de Fortalecimiento Institucional

Datos de contacto para la publicación: División de Humanidades y Bellas Artes, Departamento de Letras y Lingüística; Apartado Postal 793, Col. Centro, C.P. 83000, Hermosillo, Sonora, México. Tels.: (662) 259-21-87, Tel-fax 212-55-29. Correo electrónico: connotas@capomo.uson.mx Página web: http://www.connotas.uson.mx 


\section{Connotas. Revista de crítica y teoría literarias}

Año VII / Núm. 12 / Enero-junio 2011

COMPILAdOR DE ESTE NÚMERO

César Avilés Icedo

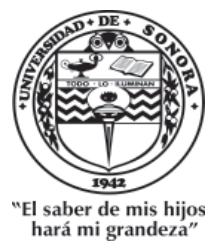

UNIVERSIDAD DE SONORA 


\section{UNIVERSIDAD DE SONORA}

RECTOR

Heriberto Grijalva Monteverde

VicerReCTORA

Arminda Guadalupe García de León Peñúñuri

Secretario General Académico

Enrique Fernando Velázquez Contreras

División de Humanidades y Bellas Artes

María Rita Plancarte Martínez

DePARTAMENTO DE LETRAS Y LINGÜÍSTICA

Martha Martínez Figueroa

COMItÉ EDITORIAL INTERNO

César Avilés Icedo

Rosa María Burrola Encinas

Fortino Corral Rodríguez

Leticia Martínez Figueroa

Jesús Abad Navarro Gálvez

Gabriel Osuna Osuna

María Rita Plancarte Martínez

DiRECTOR

Fortino Corral Rodríguez

Giuseppe Bellini

Universidad de Milán

Luis Beltrán Almería

Universidad de Zaragoza

Helena Beristáin

Universidad Nacional Autónoma de México

Raúl Bueno-Chávez

Dartmouth College

Evodio Escalante

Universidad Autónoma Metropolitana

Beatriz González-Stephan

Rice University

Aníbal González Pérez

Yale University

Aurelio González Pérez

El Colegio de México

Yvette Jiménez de Báez

El Colegio de México

Nelson Osorio Tejeda

Universidad de Santiago de Chile

\section{CONSEJO INTERNACIONAL}

Carlos Pacheco

Universidad Simón Bolivar

Rafael Olea Franco

El Colegio de México

Joan Oleza Simó

Universidad de Valencia

Julio Ortega

Brown University

Luz Aurora Pimentel

Universidad Nacional Autónoma de México

Susana Reisz

The City University of New York

José Carlos Rovira

Universidad de Alicante

Charles Tatum

The University of Arizona

Jorge Urrutia

Universidad Carlos III de Madrid

Emil Volek

Arizona State University 


\section{Índice}

\section{Artículos}

La ciudad fronteriza de Luis Humberto Crosthwaite en Estrella de la calle sexta e Instrucciones para cruzar la frontera

Martín Torres SaUchetT . . . . . . . . . . . . . . 9

Artífices y simuladores: el influjo de los medios masivos y la cultura popular en la literatura latinoamericana del siglo XX Amalia Franco Castaño . . . . . . . . . . . . . . . . . 27

“Teoría del túnel”: El pre-texto de Rayuela Margarita Díaz de León Ibarra . . . . . . . . . . . . . . 45

Metaficción hispanoamericana y crisis de la representación literaria del sujeto

Jesús Eduardo Oliva Abarca . . . . . . . . . . . . 61

Los refranes y los poemínimos: análisis de una relación intertextual

IsAbelle Pouzet . . . . . . . . . . . . . . . . . . . . . . . . 79

\section{Notas}

La ciudad como categoría estética e ideológica en Cartas de relación de Hernán Cortés y Grandeza mexicana de Bernardo de Balbuena Jesús Abad Navarro Gálvez . . . . . . . . . . . . . . . . . 99 
Hacia una hermenéutica estridentista: de la crítica romántica a la crítica de vanguardia

Alberto Rodríguez González . . . . . . . . . . . . . . . . 111

Casas de encantamiento y El espía del aire, de Ignacio Solares: la reflexión de la escritura

Alejandra Sánchez Aguilar . . . . . . . . . . . . . . . . . . 123

\section{Reseñas}

Gilberto Giménez. Estudios sobre la cultura y las identidades sociales. México: Conaculta/ITESO, 2007

Ana Lourdes Álvarez Romero . . . . . . . . . . . . . . . . . . . 135

Angélica Tornero. El mal en la narrativa de Inés Arredondo. México: Casa Juan Pablos/Universidad Autónoma del Estado de Morelos, 2008

Griselda Córdova Romero . . . . . . . . . . . . . . . . . . . . . . . 141

Guadalupe Fernández Ariza, coord. Literatura hispanoamericana del siglo XX. Historia y maravilla. España: Universidad de Málaga, 2006

Mayra Alejandra Borbón Espinoza . . . . . . . . . . . . . . 147

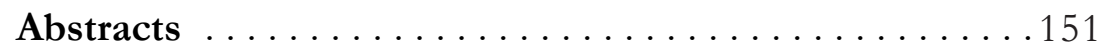

Résumés ... . . . . . . . . . . . . . . . . . . . . 157

Normas editoriales ..................... 163 
Connotas. Revista de CRíticA Y TEORía literarias/AÑo VII, NúM. 12/2011

\title{
Los refranes y los poemínimos: análisis de una relación intertextual
}

\author{
IsABelle PouzeT ${ }^{*}$
}

Resumen:

Los poemínimos surgieron tardíamente en la obra del poeta mexicano Efraín Huerta (1914-1982). Los primeros poemínimos -esos poemas muy breves compuestos de cuatro o cinco palabras- fueron publicados en 1973 en el poemario Poemas probibidos y de amor. Fueron elaborados a partir de otros textos que abarcan citas literarias, bíblicas hasta refranes pasando por eslóganes comerciales. Mediante un cambio muy leve, como la inversión de palabras o la sustitución de una sílaba por otra, el intertexto se convierte en un diminuto texto humorístico parecido al chiste o al retruécano. El humor es más palpable aún en los poemínimos que se basan en referencias populares que todos conocen. De apariencia sencilla, el poemínimo encierra cierta complejidad. Para entender sus modalidades de construcción, este artículo propone desenredar los hilos intertextuales que tejen algunos de estos poemínimos y particularmente los poemínimos basados en refranes.

Palabras clave:

Poemínimos, refranes, intertextualidad, Efraín Huerta.

*Université Rennes 2-Haute Bretagne, France. 
Los primeros poemínimos surgen en la obra poética de Efraín Huerta en Poemas probibidos y de amor publicado en 1973. A partir de allí y hasta el final de su vida, el poeta no dejará de crear estos diminutos poemas. Como lo evidencia el neologismo que los designa, los poemínimos se caracterizan por su brevedad y por su pertenencia al género poético. A nivel formal, éstos gozan de un ritmo, de rimas y se plasman en la página de la misma manera que un poema. Es más bien a nivel del contenido donde radica la dificultad para definirlos. Octavio Paz los asoció con el epigrama (287); en su nota breve dedicada a la obra de Efraín Huerta. En cambio Benjamín Valdivia los presenta no sólo como una "especie de haikú mexicano" sino también como "palabras cuasi-aforísticas, mitad paremias y mitad pensamientos extraños” (109). En el prólogo al libro que reunía todos sus poemínimos, Estampida de poemínimos, publicado en 1978, el poeta desafiaba a quien lograra definirlos:

Hacerlo, requiere de una espontaneidad diferente a la del meditado epigrama, y de un maligno toque poético que lo coloca a cien años de luminosa oscuridad del hai-kai (haikú); tampoco es un aforismo ni un apotegma ni un dogma. Para llegar o medio llegar a un acuerdo, inventé el vocablo apodogma -y todos tan intranquilos. (Huerta, Estampida 9)

Si el mismo autor nos pone ante la imposibilidad de definir sus "apodogmas", en el mismo prólogo nos deja pistas para tratar de caracterizarlos. En Poemas probibidos y de amor explicaba que Octavio Paz los había asociado con chistes, lo que había llevado al Gran Cocodrilo a regocijarse comparando este juicio con el de su hija menor, Raquel, quien los había definido de la misma manera (Huerta, Poemas 10). No cabe duda de que los poemínimos tienen como objetivo desencadenar la risa, y eso, mediante el uso de palabras disémicas como en el chiste. Éste es el primer rasgo definitorio que podemos asignar a los poemínimos.

Otro rasgo, que a diferencia del humorístico, no aparece de manera sistemática pero sí frecuente, es la intertextualidad. Gran parte de los poemínimos fue elaborada a partir de otros textos que 
abarcan citas literarias, bíblicas hasta refranes pasando por eslóganes comerciales. En su análisis retórico sobre los poemínimos, Manuel Apodaca lo había puesto de realce (1: 2-C). Benjamín Valdivia, en el capítulo de su obra El camino del fuego dedicado a la poesía de Efraín Huerta distingue diversos tipos de poemínimos: los que se construyen a base de referencias cultas, los que se construyen a base de referencias populares, los que condensan la poética de su autor y los que retoman versos de otros autores (112-25). Como lo evidencia esta clasificación, el poeta solía o bien edificar sus poemínimos a partir de referencias externas o bien verter en ellos sus propios pensamientos. Nosotros preferimos dividir en dos grupos los poemínimos: los que plasman referencias intertextuales explícitas y los que no las tienen. Dentro de este primer grupo, se distinguen los poemínimos que se basan en citas y los que fueron inspirados por refranes.

Elegimos centrarnos en este último corpus porque, a nuestro parecer, es en él donde la relación intertextual cobra una densidad máxima. Esta densidad se manifiesta mediante un doble movimiento: un movimiento que llamaremos "centrífugo" que tiende a alejar el poemínimo del texto original y un movimiento "centrípeto" que tiende a acercarlo más a él.

\section{El movimiento centrífugo: del refrán al poemínimo}

Antes de destacar el distanciamiento intertextual que se establece entre los poemínimos y los refranes, cabe recurrir a la noción de intertextualidad tal y como fue analizada por los teóricos de la hermenéutica vanguardista. Julia Kristeva explicó que "todo texto se construye como un mosaico de citas, todo texto es absorción y transformación de otro texto" (146). ${ }^{1}$ Retomando en Palimpsestes las reflexiones de Michael Riffaterre y de Julia Kristeva acerca de la intertextualidad, Gérard Genette define la intertextualidad como

\footnotetext{
${ }^{1}$ Traducción de la autora.
} 
"la presencia efectiva de un texto en otro" (8). Para él, la noción de hipertextualidad surge cuando la referencia al intertexto está declarada. Distingue el "hipotexto", o sea el texto original y el "hipertexto", el texto que se construye a partir de otros textos (13). En el caso de los poemínimos que analizamos, el hipotexto es el refrán y el hipertexto, el poemínimo. Genette destaca diferentes tipos de relaciones hipertextuales, entre ellas, la parodia. Define la parodia como "la desviación de texto con transformación mínima" (Genette 40), es decir que el objeto o sujeto del texto cambia mientras que se conserva el estilo. Es justamente lo que acontece en los poemínimos porque constatamos que la forma y el tono del proverbio no varían mientras que el mensaje que transmiten difiere del hipotexto. De ahí podemos afirmar que los poemínimos basados en refranes son paródicos.

Cabe ahora detenernos en las modalidades de la construcción paródica de los poemínimos. En el caso de estos poemas, el brote de un nuevo significado nace de una transformación del hipotexto. Esta transformación puede manifestarse tanto por una deformación como por una sustitución de una o más palabras del hipotexto. Notaremos que esta transformación formal corre pareja con una transformación semántica.

El primer grado de deformación que sufren los refranes en los poemínimos es la deformación por adición, es decir que a una palabra se le añade un morfema para alterar el significado original del hipotexto. Disponemos, por ejemplo, de los poemínimos "Saña" y "Redicho":

"Saña"

Del

Poetárbol

Caído

Todos

Hacen

Leña (Huerta, Circuito 70) 
"Redicho"
De noche
Todos los
Poegatos
Son

Pardos (Huerta, Dispersión 85)

Aquí, Efraín Huerta hubiera podido simplemente cambiar las palabras "árbol" y "gato" por "poeta" pero el juego de palabra no hubiera sido tan enjundioso. Los mots-valises presentados en estos dos poemas originan una triple isotopía. Si por un lado, se conserva el significado del refrán por la permanencia de "gato" y "árbol”, por el otro aparecen gracias a los mots-valises dos nuevos significados con la presencia del "poeta" como sujeto del poema. En el primer poemínimo se resalta la crueldad que puede reinar en el mundo literario, subrayada por el ofensivo título. En cuanto al segundo poemínimo, cabe detenernos en el significado del dicho "De noche todos los gatos son pardos" definido por el Diccionario de Autoridades como un "modo de hablar del estilo familiar, con que se explica que con la oscuridad de la noche, o falta de luz, es fácil disimular las tachas de lo que se vende o se comercia" (Real Academia Española 674). Esto nos lleva a pensar que el poema dispara agudas saetas en contra de los poetas que se toman demasiado en serio reduciéndolos a mercancías defectuosas. La tercera isotopía se percibe mediante los mots-valises que ensanchan los dos significados anteriores. En "Saña", la figura del poeta, a pesar de sufrir el rencor ajeno, está asociada con lo que simboliza el "árbol": la vida, la verticalidad, el vínculo entre la tierra y el cielo, lo cual nos desvía de las dos isotopías aludidas anteriormente. De la misma manera, la asociación del gato con el poeta no es casual: numerosos fueron los poetas románticos que relacionaron el felino con el poeta por su destreza y su finura. Notamos con estos primeros ejemplos cómo, conservando una proximidad estrecha con el hipotexto que sufre una modificación mínima, el poeta logra alejarse de su significado original. De hecho, esta relación está evidenciada por el título del segundo poemínimo, 
"Redicho", que sobreentiende que el dicho está repetido pero con otro significado.

El primer grado de deformación también se obtiene a través de una adición de una o varias letras a una palabra del refrán, lo cual modifica su significado. He aquí el poema que en lugar de título tiene la marca "(?)". Quizás sea una manera de poner en duda la certeza del poemínimo. Aparece en 50 poeminimos y dice:

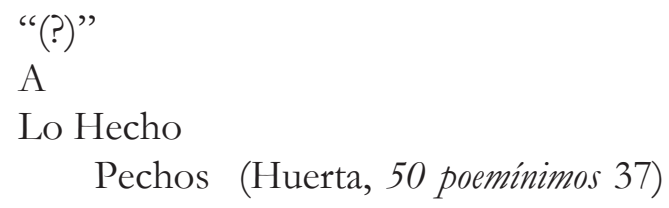

Pechos (Huerta, 50 poeminimos 37)

Con la adición del gramema de número "-s" pasamos de una sentencia seria que nos anima a aceptar las consecuencias de nuestros actos a un hipertexto de tono erótico. El poeta se apoya en la dilogía de la palabra "pecho" para alejar a su lector a leguas del significado original. Empero, éste sigue vigente porque fue empleado por su autor con su significado original en el ya mencionado prólogo a Estampida de poeminimos (Huerta, Estampida 11). ${ }^{2}$

Por tanto, a través de estos ejemplos, nos percatamos de que unos cambios mínimos colindan con un cambio semántico, el cual origina un nuevo significado sin suprimir el del hipotexto. En cambio, veremos que a partir del momento en que hay una supresión de elementos del hipotexto, el significado del refrán tiende a desvanecerse.

Ahora es necesario detenernos en el segundo grado de deformación del hipotexto. Éste ya no aparece intacto. Todavía no llegamos al grado de sustitución de palabras sino que el poeta se contenta

${ }^{2}$ En uno de sus poemínimos, Huerta había confundido una cita de Goethe con una de Heine. No corrige el error sino que lo asume: "Por ejemplo: tengo un poemínimo llamado 'Weimar', en el que a Goethe (Don Wolfango) una frase que no es suya: 'No es de Goethe sino de Heine', me aclararon, está bien. Creo que basta y sobra. Ya lo dice un poemínimo: 'A lo hecho, pechos.'” 
con hacer unas modificaciones morfológicas que se extienden a más de una palabra. Uno de los poemínimos que mejor lo plasma es el famoso "Plagio XVII" basado en "El que quiera azul celeste que le cueste" (Pérez Martínez 181):

"Plagio XVII"
La que
Quiera
Azul
Celeste

Que

Se

Acueste (Huerta, Los Eróticos 124)

El hipotexto nos prodiga un consejo: quien quiera alcanzar algo, que se esfuerce por conseguirlo. Cual un albur, este reducido poema se centra en lo que está debajo de la cintura y anima a las mujeres a tener relaciones sexuales para obtener lo que desean. El poema está dividido en dos partes, un primer momento en que aparece el sujeto de la frase designado por el pronombre "la" y un segundo momento destacado por el espacio en blanco que lo precede. No sólo este espacio, sino también el surgimiento final del juego de palabra entre "cueste" y "acueste" crean un suspenso que provoca la sorpresa del lector. El éxito de este texto radica en este efecto de suspenso y en el hecho de que "cueste" sólo sufra una prótesis y no un cambio de mayor grado. Excepto este verbo, las demás palabras que son distorsionadas son pronombres: el pronombre masculino está reemplazado por uno femenino y al pronombre complemento "le" se le sustituye uno reflexivo "se acueste". Aquí, a diferencia de los ejemplos anteriores cuyo significado seguía incluyendo el del refrán, existe entre este poemínimo y su hipotexto un abismo semántico producido por estos cambios. Notamos entonces que conforme se transforma el refrán bajo la pluma de Efraín Huerta, su significado original se esfuma.

El tercer grado de modificación de los refranes se caracteriza por la sustitución de palabras, lo cual provoca una sustitución se- 
mántica. Distinguimos dos tipos de sustitución: la que se mantiene en un nivel morfológico con el calembour o el poliptoton y la que se sitúa en el plano semántico. El poema que presenta un ejemplo de calembour es "Refraínico" sacado de 50 poemínimos:

"Refraínico"

El que

A hierro

Mata

A yerro

Muere (Huerta, 50 poeminimos 47)

En él, el autor retoma la paremia "El que a hierro mata a hierro muere" (Pérez Martínez 166) sustituyendo "hierro" por "yerro". Esta sustitución que radica en la homofonía desemboca en un nuevo significado. Ya no dice que el que emplea la violencia recibirá lo mismo, como lo define el Diccionario de Autoridades "Refrán que explica, que por lo regular se padece el daño en la misma especie que se hizo a otro" (Real Academia Española 153-154), sino que el que practica violencia muere equivocado.

Al calembour, se le añade otro modo de sustitución: el poliptoton. Con el poemínimo titulado "De Clases" tenemos un ejemplo de una sustitución por poliptoton que distorsiona el refrán "No hay peor lucha que la que no se hace" (Pérez Martínez 285):

"De Clases"

No hay

Peor

Lucha

Que la

Que no

$\mathrm{Se}$

Hizo (Huerta, 50 poeminimos 33)

De la incitación a la acción pasamos a la constatación del fracaso del ideal marxista con la elipsis "lucha de clases". El cambio 
temporal que se efectúa en el hipertexto sitúa la acción en un pasado ya terminado, lo que evidencia la imposibilidad de que se realice algún día la lucha de clases. Detrás de la carcajada que provoca el poemínimo, se divisa la decepción y el desengaño político del autor. Es de notar que el poeta se apropió del refrán para poderlo aplicar a otro contexto. Gracias al poliptoton y a la elipsis resuelta en el título del poema, se establece a nivel semántico un distanciamiento notable entre el hipotexto y el hipertexto.

El segundo tipo de sustitución sólo juega con la semántica de las palabras y ya no con su morfología. Es el distanciamiento más extenso. Podemos observar la presencia de palabras sinonímicas como en "De acuerdo" basado en el refrán "No hay que arrojarles margaritas a los cerdos" (Pérez Martínez 285):

"De acuerdo"
No hay
Que
Echarles
Cerdos
A las
Margaritas (Huerta, Dispersión 53)

La inversión de los complementos invierte también el significado del refrán. El poemínimo aconseja no dar algo malo a personas que merecen algo mejor, lo cual está reforzado por el verbo "echar" que puede ser empleado con el sentido de "echar a perder". La sustitución del verbo "arrojar" por "echar", dos verbos que comparten un significado sinonímico, nos lleva también a otro plano semántico. Si los cerdos representan a hombres sucios y groseros y las margaritas a mujeres hermosas, la palabra "echar" puede cobrar un significado sexual.

Para terminar, podemos aludir a un poemínimo cuya intertextualidad es esencialmente sintáctica porque las palabras reemplazadas no mantienen una relación semántica con las del hipertexto. Se trata de "Refrainero" que no fue publicado en ningún libro antes 
de que se hiciera la compilación de la obra de Efraín Huerta en 1988, Poesía Completa:

"Refrainero"
En la
Tierra
De
Sabios
$\quad$ El
Tonto
Es

Rey (Huerta, Poesía 561)

El doblete del refrán “ciegos/tuerto” (Pérez Martínez 192) fue cambiado por "sabios/tonto". Aunque la sintaxis del hipertexto sea la misma que la del hipotexto, la relación entre las palabras sustituidas ya no es semántica puesto que los significados de las palabras no tienen nada en común. La comicidad radica en que la lógica del hipotexto está invertida: los ineptos ya no son los ciegos sino los "sabios" y el mediocre es el "tonto". Son a los que pretenden ser sabios a quienes zahiere este poemínimo. Otra interpretación consistiría en pensar que el autor alude a los sabios mexicanos, es decir a los intelectuales, mal manejados por el "rey", es decir el presidente de México en 1978, fecha de redacción del poema.

Lo anteriormente demostrado evidencia que la parodia en los poemínimos se expresa mediante un alejamiento semántico sistemático que bien puede construirse a partir de una deformación de palabras del hipotexto o bien de una sustitución de estas palabras. Obviamente, los poemínimos más logrados son los que asocian un distanciamiento de significado con una estrechez morfológica entre las palabras hipotextuales y las hipertextuales.

Este movimiento centrífugo de alejamiento del refrán también se plasma a nivel funcional. El refrán expone una moraleja, una sentencia. Lo evidencia Luis Martínez Kleiser en el estudio preliminar a su Refranero general ideológico español "El refrán nos mira con rostro severo y nos habla con voz enronquecida y conminatoria" 
(13). En cambio, el poemínimo busca la comicidad, la cual se obtiene por la distancia más o menos extensa que existe entre él y el refrán. Conocer el refrán es necesario para captar la densidad del poemínimo, en el caso en que el lector no lo conociera, sólo lo podría tomarlo como un aforismo o un chiste. Heriberto Yépez, en su artículo "Los poemínimos: fragmentación, apropiación \& pop", en Efraín Huerta. El alba en llamas (2002), compilación de artículos críticos sobre la obra de Huerta por su hija menor Raquel HuertaNava, compara las creaciones verbales de Efraín Huerta con el arte pop (118-134). Afirma que los dos tienen en común esta voluntad de tomar un aspecto de la cultura popular para transformarlo en arte. ${ }^{3}$ Se trata entonces de una apropiación del refrán -manifestación de la cultura popular- por el arte poético. Así lo expresa Heriberto Yépez: "Utiliza la literatura para contrarrestar los refranes populares y utiliza la oralidad popular para contrarrestar la literatura." (132) Este contacto entre la cultura popular y la literatura en los poemínimos dinamita tanto la primera como la segunda. Pero de esta explosión nace una nueva percepción de la cultura oral y de la poesía. Quien participa activamente en este juego de deconstrucción y de reconstrucción es el lector. La recepción del poemínimo sería muy limitada si el lector no tuviera en mente las referencias populares a las que alude. Fenómeno idéntico se da con la recepción de los textos de minificción. Por su brevedad y la dosis de intertextualidad que pueden contener, esos textos requieren también una participación activa del lector. La reflexión de Lauro Zavala en La minificción bajo el microscopio sobre el lector de minificción bien podría aplicarse al lector de los poemínimos:

Lo que se apuesta, a fin de cuentas, es el placer cómplice de cada lectura, un placer que tal vez se prolongue más allá de ese momento, y que tal vez afecte la identidad del lector. (Zavala 56)

3 “" [...] como los artistas visuales pop, Huerta toma un icono de la cultura, lo reubica en un contexto semántico crítico, lo modifica estratégicamente y lo transforma en obra de arte." (Yépez 125) 
Así pues, "el placer cómplice" que provoca la lectura de los poemínimos se debe a la identificación de las referencias intertextuales distorsionadas. Observamos que en los poemínimos citados el movimiento centrífugo se realiza mediante el alejamiento semántico del núcleo intertextual que forma el refrán. A pesar de que el cambio morfológico suela ser leve, el poeta o bien ensancha el significado del hipotexto o bien lo elude para sustituirle un nuevo significado. Además, actúa de manera concomitante un movimiento contrario que llamamos, por oposición al primero, movimiento "centrípeto". Éste tiende a aproximar el poemínimo al refrán puesto que, en él, el poeta sigue manteniendo tanto la forma del refrán como algunas de sus características funcionales.

\section{El movimiento centrípeto: del poemínimo al refrán}

Respecto a los dos primeros grados de metamorfosis del refrán, ya notamos que la diferencia entre el hipotexto y el poemínimo a nivel formal es muy leve. Esta diferencia sigue siendo sutil cuando se trata de sustitución de palabras.

Generalmente, el sustantivo reemplazado suele compartir varios fonemas con el original, lo que crea una relación paronomástica entre ambos. He aquí el poema "Muy Cierto" calcado sobre el refrán "Hombre prevenido vale por dos” (Pérez Martínez 216):

"Muy Cierto"

Hombre

Pervertido

Vale

Por

Dos (Huerta, Dispersión 75)

La distancia entre "pervertido" y "prevenido" es muy limitada. Tienen el mismo número de sílabas y "pervertido" sigue manteniendo la rima consonante "ido" y los fonemas /p/, /v/ y /d/. 
Tenemos también varios ejemplos de paronomasias que evidencian la cercanía que subsiste entre la palabra del intertexto y el poemínimo. Por ejemplo, entre el poema "Infernal” y su refrán Efraín Huerta creó un efecto paronomástico con la sustitución de "honor" por "horror":

"Infernal"

Horror

A quien

Horror

Merece (Dispersión 66)

Cuando la paronomasia no puede ser efectiva, el autor busca mantener un vínculo con el intertexto eligiendo una palabra que tenga sonoridades comunes con la del refrán. En el caso del poema "Plagio LVIII", el verbo "casar" viene a sustituir "trabajar". Así el poeta mantiene un vínculo fónico entre las palabras gracias a la asonancia.

"Plagio LVIII"

Nadie

Sabe

Para

Quién

$\mathrm{Se}$

Casa (Huerta, Los Eróticos 144)

Ocurre lo mismo en el poema "Canción":

"Canción"

Arreolas

Somos

Y

En 
E1

Camino

Andamos (Huerta, Los Eróticos 118)

Pasamos de "Arrieros somos y en el camino andamos" (Pérez Martínez 90) a "Arreolas somos y en el camino andamos". A diferencia de los precedentes ejemplos, el autor no mantuvo el lazo morfológico con "arrieros" ya que puso en su lugar un nombre propio, el del novelista jalisciense Juan José Arreola.

Asimismo, se evidencia este movimiento centrípeto morfológico en el poema "Refrainero" aludido precedentemente. No se trata aquí de una paronomasia o de la permanencia de una sílaba, como en los poemas anteriores, sino de la sustitución por palabras que mantienen un lazo morfológico con las del refrán. En efecto, al igual que en el hipotexto los dos son sustantivos masculinos, el primero sigue en plural y el segundo en singular. A esto se suma una relación fonológica entre "sabios" y "ciegos" gracias al fonema / s / y entre "tonto" y "tuerto" gracias al fonema dental.

También los poemínimos -y no sólo los que fueron el objeto de nuestro análisis- tienden, cuando no remiten a una situación personal, a difundir una verdad. No se trata de un puro juego verbal como en ciertas parodias. Es obvio que el objetivo primero es la comicidad, sin embargo, en los poemínimos de Huerta en general se encuentran "verdades". No es que Huerta haya querido plasmar verdades -lo cual hubiera hecho con aforismos, en lugar de poemínimos- sino que la estructura misma del refrán lo impone. En efecto, si retomamos los rasgos definitorios del proverbio francés definidos por Algirdas Julien Greimas, los cuales comparten los refranes en español, notaremos que al no alejarse del hipotexto, Huerta, de cierta forma, tampoco puede separarse del propósito del refrán: trasmitir una verdad.

La presencia de lo que el crítico llama "el carácter arcaico del refrán” (Greimas 311) que se manifiesta sobre todo por la ausencia de un artículo o de un antecedente favorece la indefinición del personaje, lo cual ayuda a la identificación de quien lo escucha o lo pronuncia. Por ejemplo, si atribuimos a "Refraínico" el significado 
de "quien comete violencia muere equivocado", la ausencia de antecedente hace que cualquier lector se pueda identificar con él. Encontramos esta misma característica en el poema "Cierto" que refiere otra vez a "Honor a quien honor merece" (Pérez Martínez 216):

\author{
"Cierto" \\ Es \\ La única \\ Verdad \\ Amor \\ A quien \\ Amor \\ Merece (Huerta, Dispersión 50)
}

La permanencia de la forma original y la sustitución por la palabra "amor", la cual es muy frecuente en los dichos y los refranes, podría dar lugar a un nuevo refrán cuyo significado sería: sólo el amor se da a quien lo merece. La permanencia del presente del indicativo acrecienta la impresión de transmisión de una verdad porque el tiempo presente, como lo apunta Greimas, es un tiempo "ahistórico" que enuncia "verdades eternas" (313). Lo que diferencia el hipertexto del refrán es la presencia del título y sobre todo la afirmación introductoria "Es la única verdad". Sin embargo, este título "Cierto" y esta afirmación introductoria vienen a subrayar la idea de que lo que difunde el poemínimo no es un simple juego intertextual sino una verdad tal y como la plasmaría un aforismo. Este poema es el único poemínimo basado en un refrán que viene a resaltar el carácter gnómico del poema mediante una afirmación que lo anuncia. No obstante, éste no es el único en gozar de un título que reafirma la certeza de su discurso. Destacamos entre los poemínimos de Efraín Huerta: "Así es" (Dispersión 45), "De Acuerdo" (Dispersión 53), "De plano" (Dispersión 53), "Muy Cierto" (Dispersión 75) y "Pues sí" (Dispersión 83). Aunque no todos estos poemas presenten un mensaje de tipo paremiológico, sus títulos ponen en escena expresiones que se suelen usar en el discurso oral para 
asentar una verdad o mostrar su adecuación con la idea enunciada. De cierta forma, estos poemínimos tienden a evidenciar que ellos también, a pesar de que sean paródicos y cuyo fin principal sea la comicidad, pueden transmitir una verdad.

Otro rasgo que comparten los poemínimos con los refranes es su tono a veces agresivo. Como lo recuerda Luis Martínez Kleiser en el Refranero general ideológico español, una parte de los refranes se caracterizaba por la pulla que dirigían a ciertos grupos de la sociedad. Da el ejemplo de un refrán que se mofa de los que se pasaban el tiempo latinizando: " 'Hico, heque, hoque', pan tostado con arrope" (Martínez Kleiser 21). A través de los poemínimos que pusimos de relieve, pudimos cerciorarnos de que comparten este rasgo con los refranes. De hecho, el mismo autor ya lo había señalado en el prólogo a Estampida de poeminimos:

"Dislocar y trastocar; crear, es el único secreto de esta singular forma de expresar referencias maternales sin llegar jamás a los extremos líricos y delictuosos de la mentada por la mentada misma. (Huerta, Estampida 9)

Agredir jugando es el propósito de algunos de estos poemas. Sus blancos más frecuentes son generalmente los literatos como en los poemas "Saña" (Huerta, Circuito 70) y "Redicho" (Huerta, Dispersión 85). También el poema "Inútil" basado en el refrán "no por mucho madrugar amaneces más temprano" (Pérez Martínez 293) ataca a los que publican numerosos libros sin recibir reconocimiento:

\section{"Inútil" \\ No por \\ Mucho \\ Publicar}

Te consagras

Más

Temprano (Huerta, Dispersión 67) 
En otros poemínimos, el poeta distorsiona referencias literarias por juego y no forzosamente para agredir. Por ejemplo, el poema "Monterroseana" plasma el cortísimo cuento de Augusto Monterroso, "El dinausorio", en un ámbito sexual:

"Monterroseana"
Cuando
Desperté
La
Putosauria
Todavía

Estaba

Allí (Huerta, Circuito 68)

Estas constataciones nos llevan a interrogarnos acerca del estatuto genérico de ciertos poemínimos basados en refranes, los cuales se parecen a ellos tanto a nivel formal como a nivel de su contenido. Está claro que no se puede poner en duda el hecho de que los poemínimos pertenezcan al género poético. Si el paratexto los presenta como poemas, el lazo intertextual que mantienen con los refranes es tan fuerte que éste acaba por ensancharse y convertirse en un lazo architextual. La architextualidad, según Genette, se presenta como una "relación muda [...] de pura pertenencia taxonómica" (12). El crítico habla de relación "muda" porque sólo un paratexto puede indicar a cuál género pertenece una obra. Él explica que si no existe este paratexto, es el lector quien debe juzgar de la pertenencia genérica de la obra que lee. Esta proximidad con los refranes resulta a veces tan tenue que podríamos atrevernos a afirmar que algunos de los poemínimos basados en los refranes, en particular los que analizamos en la segunda parte, también podrían funcionar como refranes si lograran a ser usados por la gente. Aquí radica el límite de nuestro postulado: el refrán para ser "refrán" tiene que ser empleado por el pueblo, de boca en boca. Los poemínimos, si tuvieron mucho éxito por entre los literatos de la época y los estudiantes de Letras, no llegan a instalarse en la cultura popular como lo hacen los refranes. 
Esta última reflexión pone en evidencia que el poemínimo -por lo menos el tipo de poemínimo que fue el objeto de nuestro análisis- encierra en sí más potencialidad que un mero chiste y o un epigrama. Tiene el humor del chiste, goza de la brevedad y de la causticidad del epigrama, difunde una verdad como en la paremia y el dogma pero no pertenece a ninguno de estos grupos. Inclasificable, inasible, cuando apenas logramos definirlo o encontrar su origen ya se escapa para otros rumbos. No se puede definir el poemínimo pero sí se pueden analizar sus modalidades de construcción. $\mathrm{Al}$ estudiar la relación intertextual entre los refranes y los poemínimos, descubrimos que no se trata de puras imitaciones sino de verdaderas creaciones literarias. El constante vaivén entre ellos comprueba que el refrán no constituye el pretexto para inventar el poemínimo sino que el poemínimo se convierte en un pretexto para reinventar el refrán.

\section{Bibliografía}

Apodaca, Manuel. "Retórica en los Poemínimos." Excélsior 18 de junio de 1994: 2-C.

—. "Retórica en los Poemínimos (II)." Excélsior, 21 de junio de 1994: 4-C.

Genette, Gérard. Palimpsestes La littérature au second degré. Paris: Le Seuil, 1982.

Greimas, Algirdas Julien. Du sens. Essais sémiotiques. Paris: Le Seuil, 1970.

Huerta, Efraín. Poemas probibidos y de amor. México: Siglo Veintiuno, 1973.

—. Los Eróticos y otros poemas. México: Joaquín Mortiz, 1974.

- Circuito Interior. México: Joaquín Mortiz, 1977.

— 50 Poemínimos. México: Taller Martín Pescador, 1978.

- Estampida de poeminimos. México: Premià, 1980.

—. Dispersión total. México: Papeles Privados, 1986.

- Poesía completa. Comp. Martí Soler. $2^{a}$. ed. México: Fondo de Cultura Económica, 1995. 
Kristeva, Julia. Sémiotikè. Paris: Le Seuil, 1969.

Martínez Kleiser, Luis. Refranero general ideológico español. Madrid: Hernando, 1978.

Paz, Octavio. Obras Completas IV. "Generaciones y semblanzas." México: FCE, 1994.

Pérez Martínez, Herón. Los refranes del hablar mexicano en el siglo XX.

México: Conaculta y El Colegio de Michoacán, 2002.

Real Academia Española, Diccionario de Autoridades. Madrid: Gredos, 1990.

Valdivia, Benjamín. El camino del fuego. México: Gobierno del Estado de Guanajuato, 1991.

Yépez, Heriberto. "Los poemínimos: fragmentación, apropiación \& pop.” En Efraín Huerta. El alba en Ilamas, presentación y selección de Raquel Huerta-Nava, México, Conaculta/Gobierno del Estado de Guanajuato Fondo Editorial Tierra Adentro, 2002: 118-134.

Zavala, Lauro. La minificción bajo el microscopio. México: Universidad Autónoma de México, 2006. 